\title{
Review
}

\section{Pain and Pain Management in Veterinary Medicine: A Review}

\author{
Endeg Alamrew, DVM; Haben Fesseha, MVSc, DVM* \\ School of Veterinary Medicine, Wolaita Sodo University, P. O. Box 138, Wolaita Sodo, Ethiopia
}

"Corresponding author

Haben Fesseha, MVSc, DVM

Assistant Professor, Department of Veterinary Surgery and Diagnostic Imaging, School of Veterinary Medicine, Wolaita Sodo University, P. O. Box I38,Wolaita Sodo, Ethiopia; ORCID: 0000-000 I-65 I6-3036; E-mail: tseyon.h@gmail.com

\section{Article information}

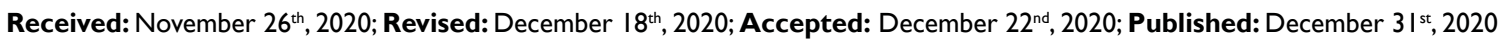

\section{Cite this article}

Alamrew E, Fesseha H. Pain and pain management in veterinary medicine:A review. Vet Med Open J. 2020; 5(3): 64-73. doi: I0.17|40/VMOJ-5-I5I

\section{ABSTRACT |}

Pain is the distress feeling, most commonly encountered in diseases, which accompanies from an early age and it is one of the important aspects of determining the level of well-being of animals. Pain is more complex in animals and it can rather difficult to determine the seriousness and impact of painful events. Hence, we have to identify the signs of pain in animals consistent with indirect markers which involve behavioral, physiological, and at last clinical responses. Moreover, specifically, the behavioral changes related to pain will be together with the overall signs also species-specific and hardly recognizable especially for an inexperienced observer seemingly unimportant which makes pain assessment even more complicated. The veterinarians have an ethical responsibility to assist in alleviate the animal pain. Although most veterinarians accept the actual fact that animals feel pain, still, post-operative pain relief isn't a routine practice in veterinary hospitals and clinics today the feeling and assessment of pain are subjective. The assessment of pain and also the documentation of the effectiveness of any interventions are fundamental principles of successful pain management. The most important aim is pain management while efforts are made to spot and treat the underlying disease and to reinforce healing and recovery. Adequate management of acute pain may additionally prevent the event of chronic pain.

\section{Keywords}

Animal; Pain assessment; Pain; Pain management.

\section{INTRODUCTION}

$\mathrm{P}$ ain is an unpleasant sensory and emotional experience beginning with a peripheral stimulus that undergoes a physiological process ultimately leading to the feeling of pain and it adversely affects the animal health and welfare. ${ }^{1}$ The quality and intensity as well as the degree of suffering from pain can only be experienced by human. However, for non-human animals, it is harder, if even impossible, to know whether an emotional experience has occurred. ${ }^{2}$ Animals, just like people, feel pain when they are injured or sick. Yet, diagnosing pain in animals is difficult since animals don't act in the same way as humans. Animals are routinely subjected to painful procedures, such as castration in cattle, tail docking in piglets, and horn removal (disbudding and dehorning) in calves. ${ }^{3}$

Painful stimuli are perceived by free nerve endings called nociceptors. Nociceptors are found in the skin, an organ of motion (periosteum, joint capsule, ligaments, muscles), the cornea of the eye, and dental pulp. Inside the body, they are also abundant in the meninges, pleura, peritoneum, and organ walls. Stimulated by biological, electrical, thermal, mechanical, and chemical stimuli the noniceptors transmit information to the brain., 4,5 These stimuli are sufficient to result in tissue injury produce a hierarchy of responses including withdrawal of the affected body part (homotopic withdrawal reflexes), changes in autonomic outflow (increased heart rate and blood pressure, depending on the species), and activation of the hypothalamo-pituitary axis, yielding increased circulating concentrations of a variety of stress hormones (e.g., prolactin, adrenocorticotropic hormone (ACTH), cortisol). ${ }^{5-}$

Pain reflects the interpretation of a nociceptive stimulus event as a sensation with highly aversive properties. ${ }^{5}$ The pathogenesis of pain perception incorporates mechanisms that result in acute or chronic pain. Pain is a recognized and common sign of potential tissue damage. Hence, pain sensation is protective in function. Though, the pathologic states of pain exist secondary to disruption of the sensitive method each peripherally and centrally or secondary to psychological conditions. It's essential to spot 
these symptoms of pain to minimize the potential tissue harm. Chronic pain is defined as pain that exceeds 3- or 6-months duration. ${ }^{8-10}$

Animal pain is unpleasant stimuli and emotional sensation that represents the animal's perception of harm or danger to the integrity of its tissues that changes the physiology and actions of the animal to decrease or avoid damage, to decrease the possibility of recurrence and to promote recovery; non-functional pain occurs when the ex-intensity or period occurs and once physiological and activity responses square measure unsuccessful in assuaging it. $^{11}$

Pain assessment and management issues have taken great attention from each health care professionals and also the public. Factors that prompted such attention represent the high occurrence of pain, continued evidence that pain is undertreated, and a growing awareness of the adverse consequences of inadequately managed pain. Physical stress responses could also be induced by degrading experiences that area unit predominantly emotional or physical or have important emotional and physical parts. It is interesting to note that the dividing lines between many of these terms are blurred, and often they are used synonymously in the literature. ${ }^{6,12,13}$

Veterinarians must become familiar with characteristic behaviors for detecting and categorizing pain in animals. It is important to note that not all animals display pain-related behavior, and it should be emphasized that this does not mean the animal is not experiencing pain. Environmental factors, species, age, body condition, and concurrent disease can influence pain behavior and should be considered when one is evaluating animals. The placebo and nocebo influence they can elicit in animals must be familiar to animal healthcare providers. In the placebo and nocebo effects, learning created by conditioning and expectations plays an important role. Both rely on a notion that conscious perception of sensory or social stimuli may act as a cue that triggers expectancy or conditioned behavior. .,6,11 $^{5}$

Veterinarians have the responsibility to promote principles for pain control in animals under their care. ${ }^{14}$ The alleviation of pain is an important area of animal welfare research. Traditionally, pain is left unnoticed in farm animals since they are assumed to be less sensitive than pets and this remains serious due to the issue of animal welfare and ethics. ${ }^{4,15}$ Hence, this review aims to enlighten the different types of pain and their assessment and management techniques in domestic animals.

\section{PATHOPHYSIOLOGY AND CLASSIFICATION OF PAIN}

\section{Pathways of Pain}

Chemical substances that modulate pain transmission are released into the extracellular tissue when tissue damage occurs. Pain receptors are stimulated by irritating the nerve endings as a result of biological, electrical, thermal, mechanical, and chemical stimuli and they transmit information to the brain. Pain perception occurs as impulses are conveyed to the spinal cord, then to the cen- tral regions of the brain. The impulses run to the dorsal horn of the spine, where they synapse with the dorsal horn neurons in the substance gelatinosa, and then enter the brain. The essential sense of pain is at the thalamus. It proceeds to the limbic system and cerebral cortex (emotional center), where the pain is felt and interpreted. ${ }^{4}$

Nociceptors are simple structures because they are at the end of the nerve fibers. There are two types of fibers: $A \delta$ and $C$, involved in pain transmission. Large A $\delta$ fibers create sharp, welldefined pain that is normally stimulated by a wound, an electric shock, or a physical blow. They are myelinated and can cause the central nervous system to move at a rate of about 20 meters/second to an action potential. Transmission is so rapid through A $\delta$ fibers that the body reacts faster than the stimulus of pain. This results in the retraction of the affected body part before the person perceives the pain. This makes a fast response: "escape" or "fight" planning. There are virtually no opioid receptors in these fibers, although pain receptors located at the ends are still on standby. There are limited possibilities for pharmacological modification of these receptors. ${ }^{5}$

In practice, using analgesic drugs, it is easy to inhibit chronic, "slow" pain, and difficult to block "sharp", "fast" pain. After the so-called first pain, the smaller C fibers transmit dull burning or aching sensations, known as a "second pain.". ${ }^{16,17} \mathrm{C}$ fibers are very thin and susceptible to damage. They do not have the myelin sheath; therefore, the conduction of painful stimuli is very slow around $0.5-2 \mathrm{~m} / \mathrm{s}$. Numerous $C$ fibers are combined in a "net" ${ }^{4,16}$; therefore, the area covered by branching C-fibres is usually broad, and the patient can locate the pain only approximately. $\mathrm{C}$ fibers react to mechanical, thermal, and chemical stimuli. They lead pain stimuli and also pruritic stimuli (which is a part of the fibers, especially sensitive to histamine). ${ }^{4,5,14}$

Patients describe pain conducted by $\mathrm{C}$ fibers as rapid, hitching, pulsing. At the ends of these nerve fibers, there are different receptors, the most important of which are the opioid receptors. The proteins forming part of these receptors are synthesized in ganglion cells and transported inside the axons, both into the synapse in the corners of the spinal cord and towards nerve endings in peripheral tissues. Inactive forms of receptors - "sleeping receptors" are incorporated in the cell membrane of nerve endings. They may be "awakened" by inflammation., ${ }^{914}$

Various cytokines produced by inflammatory cells can penetrate the damaged perineurium and activate the receptors. In this way, the opioid receptors are activated and after sensitization can react to endogenous and exogenous opioids. C-fiber nerve endings are also "sensitized" by prostaglandin and other mediators. Inhibition of prostaglandin synthesis a non-steroidal anti-inflammatory drug, and inhibition of inflammation by corticosteroids reduces the fibers nerve sensitivity and increases the pain threshold. This basic defense mechanism is based on the cooperation between the immune and nervous systems. ${ }^{10,16,18}$ As a result, pain comes in two phases. Fast-conducting A $\delta$ fibers mediate the first step and $\mathrm{C}$ fibers mediate the second half. Physiological pain has significant importance as a warning sign that ensures human safety. ${ }^{17,19}$ 
Painful states are caused particularly by tissue or nerve damage, inflammatory processes, viral infections, or demyelination and are characterized by pain hypersensitivity. ${ }^{14}$ Pain can be classified in various ways based on different criteria. Pain can be classified based on the type of damage as tissue damage like nociceptive pain, and neuropathic pain (nerve damage). The other classification of pain is based on the intensity of pain as mild, moderate, and severe. Moreover, pain is classified as acute, chronic, and cancer based on the duration of time. . $^{5,20,21}$

\section{Classification of Pain}

\section{Pain based on the type of tissue damage}

Neuropathic pain: Neuropathic pain originates within the nervous system itself and arises as a disorder of processing of nociceptive activity or as a result of abnormal activity in nociceptive pathways. ${ }^{22}$ Damage to a nerve or some other part of the central nervous system results in neuropathic pain. This type of pain is frequently not diagnosed in veterinary medicine, mainly because animals cannot communicate a problem such as a tingling sensation. Typically, neuropathic pain is manifested by disproportionate stimulus hypersensitivity (hyperalgesia), an irregular feeling of pins and needles (hyperpathia), and nociceptive reactions to harmless stimuli (allodynia). ${ }^{6,7}$

Nociceptive pain: Nociception is a term used to describe the physiological (physiological pain) response caused by high-intensity stimuli (e.g. acute withdrawal; autonomous outflow changes). Nociceptive pain is a medical term used to describe pain caused by physical injury or possible damage to the body. Examples might be the pain felt from a sports injury, a dental procedure, or arthritis. ${ }^{7,23}$ Nociception is the process by which the unpleasant noxious stimulus from the periphery is transmitted through the spinal cord and to various areas of the central nervous system resulting in the physiological sensation of pain and associated negative emotional response and memory, ultimately results in the sensation of pain. ${ }^{10}$

Nociceptive pain is the feeling of warning against the danger of tissue damage or indicating already inflicted damage as a result of illness or injury. Pain receptors may come from external tissues-integuments, skin, and mucosal pain -from internal organs (eye, ear, nasopharynx, heart, blood vessels, abdominal organs, and pelvis minor). There is also a pain in bones, joints, and muscles. Incentives that irritate pain receptors are chemical and physical stimuli that function outside of the nervous system. This stimulus is converted into electrical impulses conducted by the physiological undamaged nervous system to centers where they are recognized as pain. They go to the reticular system whereas a non-specific impulse-stimulates cerebral cortex activity and reaches the limbic system, which determines the emotional response to pain. Defense reflex is formed in the brain centers. ${ }^{4,6}$

\section{Pain based on the duration of time}

Acute pain: A psychophysical experience triggered by high intensity, potentially tissue-damaging stimuli or peripheral nerve damage is acute pain. ${ }^{5}$ Once, acute pain was clearly characterized in terms of time. It is now seen as a dynamic, painful experience with emotional and cognitive as well as sensory; characteristics that occur in response to tissue trauma. ${ }^{7,24}$ Acute pain soon disappears once the damaged tissue has been healed. In contrast, chronic (or persistent) pain lasts beyond the expected healing time for injured tissue and can be more difficult to recognize because it is not possible to identify behavior that would uniquely and reliably indicate the existence of chronic pain. ${ }^{1,9}$

Acute pain is associated with spasms of the skeletal muscle and stimulation of the sympathetic nervous system, induced by a particular illness or injury, serves a useful biological function, and is self-limited. Both the peripheral and central nervous system elements of the pain transmitting pathway demonstrate tremendous plasticity, strengthening pain signals, and creating hypersensitivity in assessing a chronic injury. When plasticity favors protective reflexes, it can be beneficial, but a chronic pain condition may result when the changes persist. ${ }^{4}$

Trauma, surgery, labour, medical treatments, and acute disease conditions are common causes of acute pain. It is also important to realize that various tissues and organs of the body can have different sensitivities to painful stimulation. For example, mucous membranes, cornea, or dental pulp are considered to be extremely sensitive, whereas parenchymatous organs are characterized as less painful..$^{25,26}$

Chronic pain: Chronic pain was once defined as pain that extends 3 or 6-months beyond the onset of the expected period of healing. ${ }^{9}$ New meanings, however, distinguish chronic pain from acute pain based on more than just time pain is now known as pain that persists past the recovery period, with levels of defined pathology that are often poor and inadequate to justify the pain's existence and/or duration. It persists for longer than the expected time frame for healing, or it can be associated with a progressive noncancerous disease, such as osteoarthritis. ${ }^{8}$

Chronic pain can result from psychological states, has no biological purpose, and has no end-point that can be differentiated. Persistent pain associated with injury or diseases (diabetes, arthritis, or tumor growth) can result from changes in the properties of peripheral nerves. This can occur through damage to the nerve fibers, leading to increased spontaneous firing or alterations in their conduction or neurotransmitter properties. The use of topical and even systemic local anesthetics to treat various neuropathic pain disorders (such as postherpetic neuralgia) is likely to indicate their action on sodium channels that accumulate in weakened nerve fibers. $^{1,4}$

Cancer pain: Cancer pain is the result of primary tumor growth, a spreading cancerous disease, or the toxic effects of chemotherapy or radiation. "Malignant pain" or "cancer pain" is also called pain associated with potentially life-threatening conditions such as cancer. In this monograph, the word "cancer pain" is used for the sake of brevity. Cancer pain involves pain caused by the disease itself (e.g., tumor) invasion of tissue compression or penetration of nerves or blood vessels, organ obstruction, infection, inflammation, and/or painful diagnostic or treatment procedures (e.g., 
biopsy, postoperative pain, and chemotherapy or radiation therapy toxicity). ${ }^{5,27}$

Overlooked or unrecognized source of pain: Unintentional pain or discomfort associated with veterinary procedures is easily overlooked. Procedures that might cause discomfort or pain include intravenous (IV) catheterization, ear cleaning, manual stool evacuation, and anal sac expression (especially in cats). Assess in-hospital procedures where there might be unintended discomfort and minimize or remove those causes. To reduce the pain or discomfort of certain treatments, use an opioid if appropriate. If an animal is to be confined or treated unnecessarily because of its agitation, hostility, or pre-existing discomfort, use anxiolytics, sedation, and/ or anesthesia to combat, subsequent pain or damage, and aversion when appropriate. ${ }^{28}$

Conditions in which the amount of pain endured by animals is uncertain include many visceral, gastrointestinal, and urogenital diseases; disorders of the central nervous system; and dermatological disease. Specific management for pain conditions and procedures may include pharmacologic and/or non-pharmacologic management or simply a different or more careful approach to handling the animal. Any treatments and disorders that are generally known as causing discomfort in dogs can be over-exposed in cats. Osteoarthritis, intervertebral disc disease, and spondylosis, for instance, are common in older cats, and yet many of the behavioral changes associated with these diseases have been attributed to old age rather than pain. ${ }^{29,30}$

Cats and dogs with behavior issues also have an underlying medical condition that can be painful. For example, the cat that urinates inappropriately may have a painful lower urinary tract disease. Pain management plays an important role in the animal's care in these types of situations. Besides, there are different types of overlook pain in different animals that are listed below in Table 1.

\section{BEHAVIORAL AND PHYSICAL SIGN OF PAIN IN ANIMAL}

In most developed countries, pain is the most common explanation for physician consultation. It is a major symptom in many medical conditions and can interfere with the animal quality of life and general functioning and it shows the following signs. ${ }^{13,25}$

\section{Vital Sign of Pain in Animal}

Vital sign scales do not distinguish between causes of pain, such as surgical pain or another cause. Besides, vital signs may be normal in animals experiencing chronic pain. These indicators are not specific enough to distinguish pain from other stresses such as anxiety, fear, or physical responses to certain medical conditions (such as anemia). In other words, an animal can still be in pain even if its heart rate and breathing rate are normal. Vital signs of pain in animals such as heart rate, respiratory rate, and blood pressure may be used to assess responses to an acute painful stimulus, particularly during surgery or after severe trauma. Measurement of vital signs can also be used to assess pain in some situations such as horses with colic. ${ }^{8}$

\section{Behavioral Sign of Pain in Animal}

A systematic and holistic approach that considers the animal, as well as its environment, is essential to recognize changes in behavior and physiologic parameters. Behavioral signs of pain, including both loss of normal behavior and development of new and abnormal behaviors, may be subtle and easily overlooked by both the owners and the veterinary health care team. ${ }^{12}$ The expression of pain through behavior has many dimensions, including suppression of behavior as well as active indicators of pain for example terminal stages of some abdominal disorders can produce severe depression with very few outward signs, and animals severely debilitated by major surgery may be the least able to express pain overtly. ${ }^{6,33,34}$ Agreed throughout the literature is the lack of obvious pain expression by the animal and a higher tolerance level or our current inability to interpret fully the subtle behavioral changes being presented. A similar probe exists with wild or semi-feral in different species, where non-disclosure of pain is a valuable survival strategy for animals subject to predation in a wild state..$^{20,25}$

Changes in normal animal behavior are almost always associated with pain or discomfort. Knowledge of the animal's normal behavior is required to optimally characterize subtle abnormalities. The history (e.g., activity, personality changes) and the owner's assessment are valuable aids. Common behaviors displayed by animals in pain include the following: anxiety, depression, inappetence, reluctance to move, reclusive (non-interactive), irritable-aggressive. ${ }^{5,35}$

Often behavioral indicators are used as guidance for detecting the presence of pain, rather than an accurate assessment of its severity. Indications of pain severity could have particular relevance for monitoring the progression of a condition assessing the

\begin{tabular}{|llll|}
\hline Table I. Frequently Overlooked Pain & \multicolumn{1}{c|}{ Definition } & \multicolumn{1}{c|}{$\begin{array}{c}\text { Clinical } \\
\text { Manifestation }\end{array}$} \\
\hline Pain Type & & $\begin{array}{l}\text { Somatic pain originates in the skin and is called Superficial } \\
\text { pain. If it originates in the muscles, Bones, joints, or connective } \\
\text { tissues it is called deep pain. }\end{array}$ & $\begin{array}{l}\text { Pallor } \\
\text { Sweating } \\
\text { Nausea }\end{array}$ \\
\hline Visceral pain & $\begin{array}{l}\text { Visceral pain originates in internal organs. These nociceptors } \\
\text { are activated by actual tissue damage, or in the absence of } \\
\text { damage, pressure, and stretch of the organ. }\end{array}$ & $\begin{array}{l}\text { Headaches movement disorders } \\
\text { Weakness }\end{array}$ \\
\hline Bone pain & $\begin{array}{l}\text { It is usually deep, penetrating, or dull. It commonly results } \\
\text { from injury. Other less common causes of bone pain include } \\
\text { bone infection (osteomyelitis), and tumors. }\end{array}$ & $\begin{array}{l}\text { Swelling and tenderness near the affected area } \\
\text { and Fatigue }\end{array}$ \\
\hline & & 25 \\
\hline
\end{tabular}


efficacy of analgesia and decision-making on animal endpoints for research and clinical practice. The advantage of using behavioral indicators is that changes in behavior are immediate in their appearance, unlike physiologic indices, which take time to quantify. ${ }^{13}$

Besides, it has been revealed that changes in behavioral patterns correspond well with physiologic signs of distress in various farm animals. To get a reliable assessment of pain it is important to describe in full the increase and decrease of complex behavior patterns elicited by noxious stimuli and to identify subclasses of particular behaviors. ${ }^{7,36}$

Various other factors also need to be considered when using behavior as a pain indicator. The social context of the animal is an important factor. An early symptom of pain may be alienation from group members. The presence of more than one behavioral indicator of pain (postures, facial expressions, stereotypical movements, vocalizations) helps to confirm pain. Similarly, the behavioral indicators of pain may be related to concomitant bodily changes, both external (morphologic changes, body weight) and internal (hormonal responses, brain activity. ${ }^{9,15}$

There are other indications of well-being that suggest positively the absence of pain, distress, or discomfort. In pigs, for example, some signs of contentment include play (including playful fighting, nudging and running, nibbling at the bedding, or grabbing and biting chains or other toys) vocalization (including conversational grubbing-up inside groups or houses and the characteristic grubbing-up of sows calling their piglets to suckle or stimulate suckling) and good health along with good growth and condition. ${ }^{4,37,38}$

\section{Physiological Indicator of Pain}

Pain can be assessed using a change in different physiological parameters such as a change in heart rate, heavy breathing, enlarged pupils, and slowed reflexes. The main glucocorticoid hormone that is released in response to stress including pain is cortisol. The corticosteroid level can be measured in plasma or saliva and is a widespread means for the physiological assessment of the activity of the hypothalamus-pituitary-adrenal axis (HPA) which is activated in painful conditions. The measuring of cortisol has been used in animals to estimate the effects of different painful procedures such as abdominal surgery, electroimmobilisation, and castration.,7

Physiological signs such as increased breathing and increased blood pressure in the heart rate or dilated pupils can be symptoms of pain or stress, but should not be relied on as the only pain indicators. ${ }^{25,39}$ Cardiovascular characteristics and endocrine measures are frequently analyzed for significance and correlation with pain severity.

\section{Heart Rate}

Veterinarians have stated heart rate as the primary parameter used to assess pain intensity in clinical cases, along with their subjective assessment of demeanor. They have significant differences in animal heart rate that have been found between pain and control groups in wound sensitivity or peri-operative analgesia studies..$^{30,38}$

However, found heart rates and plasma cortisol to be significantly higher in post-operative exploratory colectomy cases compared to controls throughout the period. This was associated with the significant differences in pain scores seen between case and control groups, although no correlations could be drawn. ${ }^{15,38,40}$

\section{PERCEPTION AND ASSESSMENT OF PAIN IN ANIMALS |}

It is a challenge to understand the pain in animals since animals do not interact in the same way humans do. However, some speciesspecific behaviors can indicate pain and help us recognize it. For example, animals that are natural predators, such as dogs, behave differently when in pain than do prey animals, such as rabbits and horses. ${ }^{15}$ In these species, prey animals prefer to conceal their discomfort, making it much more difficult to recognize pain. Subsequent discussions will explore methods that promote evaluation and resolve pain reassessment. Special consideration should also be given to the evaluation of a patient with chronic pain, particularly chronic non-cancer pain. Related neural remodeling (central sensitization) suggests that without an obvious physical trigger, the pain - exists. The clinician must avoid attributing the pain to psychological factors in such situations and acknowledge and support the patient's self-report of pain., ${ }^{6,38}$

Recognition and assessment of pain have always been an integral part of animal care and veterinary clinical practice, but clinical research in this aspect of animal welfare has grown dramatically in the past 20 -years. ${ }^{26}$ At least in part, this new focus is possibly due to societal demands for pain relief both in ourselves and in our pets. Interest in animal pain and welfare is closely tied to the increased public awareness of issues surrounding the use of animals in biomedical research. The ethical mandate to justify the value of animal research and to guarantee that every effort is made to limit pain and distress codified in the Animal Welfare Act and its subsequent amendments drive the planning, review, and execution of most biomedical research using live animals. ${ }^{10,28}$

Pain assessment is at once one of the most difficult and important tasks. It has a crucial role in understanding the basic concepts of pain and to produce a proper clinical diagnosis, and to determine the effectiveness of medication or other means of intervention. Assessing behavior is an integral part of the historytaking and physical examination of any animal. To detect pain and choose an effective intervention, knowing normal behavior is crucial. The input of the owner is invaluable in determining abnormal behavior that may be linked to pain. ${ }^{26}$

Systematic assessment of pain using defined and validated pain scoring systems or scales will help to improve the recognition and treatment of painful conditions in horses. In addition to raising awareness of such pain states, they may enhance agreement between different observers or caregivers on the amount of pain a horse is experiencing, thus providing a reliable record of pain severity over time. For a pain scoring system to reliably work in prac- 
tice, it should be easy to use, with relevant well-defined parameters that can be assessed repeatedly and quickly by different observers with consistent results. ${ }^{41,42}$

\section{Pain Scale}

A pain scale tests the pain severity or other characteristics of a patient. One device that veterinarians may use to rate the discomfort of an animal is a pain scale. A pain scale is a questionnaire that includes the following characteristics: species, breed, age, gender, environment and rearing conditions, cause of pain (such as trauma or surgery), body region affected (such as the abdomen or muscle), and the duration and intensity of pain. Pain scales that consider species-specific behaviors are likely to be more accurate than generic scales that rely heavily on subjective assessment and interpretation. ${ }^{29,43}$

Pain scales in medical contexts are a common coping method and are used in a number of medical settings. Pain scales are a necessity to assist with a better assessment of pain and patient screening. Measurements of pain help to assess the severity, type, and duration of pain and are used to make an effective diagnosis, determine a treatment plan, and evaluate the efficacy of treatment. In medical settings, accurate pain measurement is a necessity, especially if the pain measurement is to be used as a screening tool, either for possible diseases or medical conditions or as a form of triage to assess the urgency of one patient over another. ${ }^{33,44}$

The use of pain scales to record pain intensity is well established for animal patient patients ${ }^{45}$ and is becoming more common in veterinary medicine ${ }^{26,46}$ it should be noted that formal scale construction and thorough clinical validation has not been pursued in most equine pain scales. The pain scale should be accurate enough to detect mild, moderate, or extreme pain, preferably have a linear relationship to pain severity, and should be validated and unique to the type of pain being measured. ${ }^{35,45}$

To evaluate the intensity, visual or analog scales are used to compare pain with the strongest pain which the patient ever suffered. In practice, the most popular scale divides pain into very strong, strong, moderate, weak, and no pain. The visual analog scale is used to assess pain by an increasing score: from 0 -meaning no pain, to 10-meaning the strongest pain endured in life. An observable feature that makes a distinction between acute and chronic pain is the length of pain. Relapses can occur after an acute period of back pain, and with them, acute pain becomes chronic pain. Pain that lasts longer than three months is chronic pain and it can be continuous and paroxysmal, as in headaches or neuralgia.,45

\section{Responses to Pain}

Animals may respond to pain in ways, excessive activity, or lethargy. Like humans, animals also exhibit various signs when in pain. the absence of normal behavior the most striking sign of pain in animals, which is why it is essential to be familiar with the normal behavioral pattern of the species. Normal behavior may include various species-specific actions such as head pushing of goats and general activity and vocalization of pigs. Although there are indi- vidual and species variations, some common signs that an animal is in pain include changes in behavioral patterns appearance, posture, gait, appetite, response to handling, and weight. ${ }^{30,47}$

Signs of acute and chronic pain may differ. In severe acute pain, animals may have signs of anxiety, changes in eye expression (including fixed and dilated pupils or tearing), restlessness, lameness, changes in appetite, changes in behavior, increased or decreased physical activity, self-mutilation, and vocalization. In chronic pain, responses include guarding behavior in movement and posture, avoidance of pain-aggravating influences, seeking pain-relieving factors and environments, self-care of a painful region, and signs of stress. Some of the signs in chronic distress states may include hirsutism, weight loss, inappetence, and an indefinable "unhappy eye expression., ${ }^{4,9,35}$

During the assessment of pain, the location, intensity, duration, and quality of pain are the main clinical features that should be considered. These qualities are evaluated mainly subjectively. ${ }^{46}$ The location of pain allows the determination of the possible cause. The location of pain does not always correspond to the site of injury or disease process. Deep organ pains are particularly poorly located. This is clinically important as it can hinder the location of the disease. Pain also happens as a phenomenon that represents pain (projected). The projection hypothesis derives from the fact that there are no pain receptors in the inner organs, only the overlying peritoneum has substantial sensory innervation. The pain severity encountered by the patient is individual and the most difficult attribute to measure. Its tolerance is the exponent of pain severity. Women have the highest tolerance, men, and children at least. ${ }^{1,15,48}$

\section{PRINCIPLE OF PAIN MANAGEMENT}

Pain management in veterinary medicine is a routine part of the physical examination and treatment plan. Pain and the types of behaviors associated with it are good indicators of a physical problem. The type and extent of the pain can help the veterinarian diagnose the problem and select the appropriate treatment. Good pain management can help a sick or injured animal recover faster. ${ }^{7}$ Ongoing reassessment and revision of pain control, titrating medications up or down to meet patient needs, should be accompanied by original pain management. Anticipate that after discharge from the hospital, increased symptoms of pain will occur, such as when residual sedation or a local anesthetic wears off after the patient has returned home. In addition, when hospitalized, some animals can mask behavioral signs of pain, and signs of pain become visible only at home. The client should be counseled about this contingency, and pain medications should be made available., ${ }^{1,6}$

Acute surgical, traumatic, and disease-related pain is generally treated with one or more analgesic, or pain-relieving, drugs. The selection of the most effective medication or drug combination is based on the predicted pain severity, the overall health of the pet, and the particular medications that are beneficial for the species. The advantage of the pain management regimen can be maximized by reducing the discomfort of your pet and ensuring all-round good care. ${ }^{1,12}$ 
The person should be adapted to the living conditions, diet, and degree of contact with other animals and people. For instance, under normal circumstances, separating a pair of dogs that enjoy playing vigorously together might be traumatic for the dogs. But it's less painful for the recovering dog to temporarily separate the dogs after one has had surgery (to allow the incision to start healing) if the human caregivers spend enough time engaging with it. A combination of good nursing care, non-drug methods (for example, bandaging, ice packs or heat, and physical therapy), and drug therapies are needed to handle animals that are under stress and in pain. Management of pain is broadly categorized into pharmacologic and non-pharmacologic (non-drug) methods. ${ }^{25}$

Multimodal approaches should be practiced in the treatment of pain. Pharmacological and non-pharmacological treatment approaches, physical therapy, neuromodulation, psychological interventions, and in some instances, intrusive techniques are available. It is extremely important to ensure mindful and comprehensive care for the patient and to clarify and obtain acceptance of the chosen method of treatment from the patient. ${ }^{4,22}$

\section{Pharmacologic Treatment}

The primary method of pain treatment is pharmacotherapy. The World Health Organization (WHO) developed and adopted pain control guidelines in 1986, called the WHO scheme or the threestage analgesic ladder. It has become the standard for analgesic treatment globally. Although it refers to the treatment of cancer pain, it is also commonly used to treat chronic pain with different substrates. There should be immediate oral administration of medications in the following order if pain occurs: nonopioids (aspirin and paracetamol); then if necessary, moderate opioids (codeine); followed by heavy opioids such as morphine until the patient is pain-free. ${ }^{4-6}$

Pharmacologic treatment is the mainstay of pain therapy. Almost half of the individuals who suffer from pain choose a nonprescription analgesic as their initial choice for pain relief. The chosen route of administration can have a profound impact on the pace and efficiency with which the medication operates. Routes of medicine entry into the body include enteral, sublingual, buccal, intravenous, subcutaneous, pulmonary, and transdermal. The absorption, distribution, metabolism, and excretion of analgesic medicines is a series of complex processes that vary with each individual. ${ }^{6,25,49}$

The choice of medications should be based on an effective diagnosis and an analgesic medication commonly used. It is important to take into account possible side-effects that occurred during the previous use of the drugs. It is also important to take into account the possible interaction of the proposed drug with other medicines used by the patient for other diseases. To obtain effective pain control, a combination of drugs with different mechanisms of action are used. They are also available in the form of ready-prepared formulations containing a combination of two or more components. ${ }^{1,4}$

\section{Opioid}

Opioids (such as morphine) are used mainly by licensed practitioners for pain-relieving purposes despite certain wellknown side effects. In many different animals, particularly cats and dogs, these drugs are the most powerful analgesics available for the treatment of acute pain. By working on the central nervous system, opioids relieve pain. Their side effects, such as sluggishness, change in mood (good or bad), and excitement, are related to other central nervous system processes. Opioids are used to treat moderate to severe pain that does not respond to non-opioids alone. They are often combined with non-opioids because this permits the use of lower doses of the opioid (i.e., dose-sparing effect). Nearly all types of pain respond to opioids; however, nociceptive pain is generally more responsive to opioids than neuropathic pain. ${ }^{15,50}$

\section{Non-steroidal Anti-inflammatory}

For many animals, non-steroidal anti-inflammatory drugs are useful as part of a pain management regimen after surgery or to relieve chronic pain, such as that caused by osteoarthritis. Some medication examples in this class are carprofen, firocoxib, and meloxicam. NSAIDs work by reducing inflammation, one cause of pain. The primary mechanism of action of non-steroidal antiinflammatory drugs is inhibition of the enzyme cyclooxygenase which blocks prostaglandin synthesis. Acetaminophen, another non-opioid, appears to act mostly via a central mechanism. ${ }^{91,1422}$

It has a variety of useful functions, including defense of gastric mucosa, maintenance of renal sodium and water balance, and platelet aggregation. In some post-operative scenarios, such as the hypovolemic or dehydrated patient, renal function may be dependent on prostaglandin synthesis. Even the brief administration of nonsteroidal anti-inflammatory drugs (NSAIDs) may impair renal function, but there is no known predictor of nephrotoxicity. ${ }^{1,51}$

\section{Local Pain Relief}

Local anesthesia can be defined as the reversible blockade of nerve conduction by regionally applied agents, for sensory ablation, which is the removal of sensation and pain. Depending on the agent used and where the agent is applied, both the motor and sensory nerves can be blocked. There is wide variation in medicines used with differing potency, toxicity, and duration of action. ${ }^{48,49}$

Only the region surrounding a wound or surgical site is numb with local anesthetics (such as lidocaine). In large animals, local anesthetics are often used in surgeries either alone or together with other types of anesthesia to minimize the potential risks and complications of general anesthesia. In small animals, local anesthetics are usually used for minor procedures such as suturing of cuts. Using local anesthetics before surgery may allow veterinarians to reduce the amount of more powerful general anesthetics needed during longer and more complex surgical procedures. A local anesthetic is another type of adjuvant analgesic. These drugs block sodium channels and inhibit the generation of abnormal impulses by damaged nerves to exert their peripheral 
analgesic effects. ${ }^{40,45}$

\section{Non-pharmacologic Treatments for Pain}

No pharmacologic treatment should supplement, but not replace, the use of medications. Many different vitamins, herbal preparations, nutraceuticals, and natural remedies are available. Most of these therapies have not been tested in scientific trials, so they are not guaranteed as safe and effective. Some may prove neither helpful nor harmful, a few might be helpful, and others might be harmful. If you are interested in giving your pet a nutritional or herbal supplement, consult your veterinarian to make sure it will not interact with any other drugs your pet is taking. ${ }^{25,52}$

\section{Surgical Approaches}

Most pain can be managed by simple non-invasive methods. Often, however more invasive methods are needed, including surgery. Orthopedic approaches to pain management include both nonsurgical (conservative) approaches and various surgeries. ${ }^{53}$

\section{Neuromodulation}

Neuromodulating treatments are aimed at stimulating the pain systems. Currently, several neuromodulation methods are used: percutaneous nerve electrostimulation (TENS), peripheral nerve stimulation, acupuncture, and vibration. Neuromodulation supports pain treatment methods and activating the pain inhibitory mechanisms can reduce pain and improve the quality of life of patients with chronic pain. ${ }^{4-6}$

\section{Psychological Therapies}

Psychological factors have a significant impact on the perception of pain and the efficacy of treatment. All patients with chronic pain should also be able to benefit from competent clinical support, which can impact the emotional component of pain. Among the psychological methods that can be effective as a technique supporting the treatment of chronic pain, the most commonly used are cognitive therapy, behavioral therapy, relaxation techniques, and hypnotherapy. ${ }^{4}$

\section{Invasive Methods}

Invasive pain control approaches should be incorporated and applied in particular situations by experienced clinicians. There are many methods: from individual nerve blocks, by intrathecal administration of drugs to neurodestractive methods (thermolesion, neurolysis) and neurosurgery. More and more approaches to relieve pain are provided by modern medicine. This makes it possible to bring relief to people suffering from various ailments. ${ }^{1,5}$

\section{Physical Rehabilitation and Physiotherapy}

Physical therapy and rehabilitation is a supporting method used in the treatment of pain. Thermotherapy (heat), cryotherapy (cold), laser therapy, electrotherapy, manual therapies, medicinal extracts, kinesitherapy, are the most common methods of physical care. These methods are used appropriately and may improve the life and mobility of some patients. ${ }^{4}$

Physical rehabilitative methods of pain management are appropriate for many types of pain and are essential in patients with chronic non-cancer pain. In addition to relieving pain, such methods can reduce fear and anxiety, improve physical function, and alter physiological responses to pain. Stretching, exercise/ reconditioning (to enhance stamina, agility, and flexibility), gait and posture preparation, and attention to ergonomics and body mechanics are therapies used in physical rehabilitation. Thermotherapy (heat application), cryotherapy (cold application), counter-irritation, and electro analgesia are other non-invasive physical remedies for pain (e.g., transcutaneous electrical stimulation). ${ }^{45}$

Usually, physical (sensory) procedures are patient-specific and inhibit nociceptive feedback and pain per experience. Some measures that can reduce pain intensity and improve the patient quality of life such as massage, positioning. ${ }^{54}$

\section{Massage}

It is a process of rubbing and kneading parts of the body, particularly the joints and muscles with the hands, to alleviate pain and reduce stress. Massage can interrupt the patient's cycle of distress. It can increase blood circulation as well as lymphatic circulation. Massage can also initiate an analgesic effect on the area being rubbed and decrease inflammation and edema. In addition, it can release muscle spasms manually while increasing the release of endogenous endorphin and opposing sensory stimuli that bypass pain signals. ${ }^{55}$

The body's soft tissue rubbing and kneading process will contribute to the relaxing of the stressed muscles, increase the flow of blood to the underlying tissues, and relieve pain. The exact mechanism of pain reduction in massage is still unknown; however, there are some studies and expert's hypotheses that suggest that the process of massage can lead to an increase in dopamine levels which decreases pain. In addition to that, massage can lead to the relaxation of the muscle tension that often arises when pain presents. ${ }^{54}$

\section{CONCLUSION}

The experience of pain is a highly complex phenomenon with physical, behavioral, cognitive, emotional, spiritual, and interpersonal aspects. This multidimensional nature of pain must be acknowledged in the assessment and management of patients. Painful states are caused particularly by tissue or nerve damage, inflammatory Offering and providing adequate pain management enhances the patient quality of life, improves the humane animal bond, encourages the team, and benefits the practice. The comprehensive study of behavior can provide access to subjective experiences and assessment of pain in different animals. Attempts to rank and score behaviors to assess pain severity have been undertaken, with some promising results being 
published. Behaviors specific to regional or type of pain have been observed, quantified, and validated by a range of treatment and analgesic approaches. However, accurate and localized detection of pain in any animal is notoriously difficult. As described above there are now methods for assessing pain in animals which could lead to more effective pharmacological or on-pharmacological pain management and substantial improvement in animal welfare. For acute pain, medications are provided to provide analgesia and reduce the occurrence of chronic pain. Different pharmacological and non-pharmacological approaches should be considered when prescribing before administration.

\section{CONFLICTS OF INTEREST}

The authors declare that they have no conflicts of interest.

\section{REFERENCES}

1. Mathews K, Kronen PW, Lascelles D, Nolan A, Robertson S, Vm Steagall P, et al. Guidelines for recognition, assessment and treatment of pain: WSAVA Global Pain Council members and coauthors of this document. I Small Anim Pract. 2014; 55(6): E10E68. doi: 10.1111/jsap.12200

2. Wright A. A criticism of the International Association for the Study of Pain (IASP's) definition of pain. Journal of Consciousness Studies. 2011; 18(9-10): 19-44.

3. Walker KA, Duffield TF, Weary DM. Identifying and preventing pain during and after surgery in farm animals. Applied Animal Behaviour Science. 2011; 135(3): 259-265. doi: 10.1016/j.applanim.2011.10.021

4. Świeboda P, Filip R, Prystupa A, Drozd M. Assessment of pain: Types, mechanism and treatment. Ann Agric Environ Med. 2013; Spec no. 1: 2-7.

5. Wiese AJ. Assessing pain: Pain behaviors. In: Handbook of Veterinary Pain Management. NX Amsterdam, The Netherlands: Elsevier Publications; 2015: 67-97.

6. Gaynor JS, Muir W. Handbook of Veterinary Pain Management. $3^{\text {rd }}$ ed. Oxford, UK: Elsevier Health Sciences; 2014.

7. Landa L. Pain in domestic animals and how to assess it: A review. Vet Med. 2012; 57(4): 185-192. doi: 10.17221/5915-VETMED

8. Richmond M. Should pain be the fourth vital sign? Vet Nurs J. 2016; 31(8): 249-252. doi: 10.1080/17415349.2016.1192966

9. Shaffran N. Pain Management for Veterinary Technicians and Nurses. NJ, USA: John Wiley \& Sons; 2014.

10. Dinakar P, Stillman AM. Pathogenesis of pain. Semin Pediatr Neurol. 2016; 23: 201-208. doi: 10.1016/j.spen.2016.10.003

11. Maria Eugênia AC, João Augusto RB, Júlio Otávio JB. Attitudes of cattle veterinarians and animal scientists to pain and painful pro- cedures in Brazil. Prev Vet Med. 2020; 177: 104909. doi: 10.1016/j. prevetmed.2020.104909

12. Anil SS, Anil L, Deen J. Challenges of pain assessment in domestic animals. J Am Vet Med Assoc. 2002; 220(3): 313-319. doi: 10.2460/javma.2002.220.313

13. Breivik H, Borchgrevink PC, Allen SM, Rosseland LA, Romundstad L, Breivik Hals EK, et al. Assessment of pain. BJA: British Journal of Anaesthesia. 2008; 101(1): 17-24. doi: 10.1093/bja/ aen103

14. Viñuela-Fernández I, Jones E, Welsh EM, Fleetwood-Walker SM. Pain mechanisms and their implication for the management of pain in farm and companion animals. Vet J. 2007; 174(2): 227239. doi: $10.1016 /$ j.tvj1.2007.02.002

15. Kitchell RL, Erickson HH. Animal Pain: Perception and Alleviation. NY, USA: Springer; 2013. doi: 10.1007/978-1-4614-7562-0

16. Żylicz Z, Krajnik MJ. Jak powstaje ból? Neurofizjologia bólu dla początkujących. Pol Med Paliat. 2003; 2(1): 49-56.

17. Helms J, Barone C. Physiology and treatment of pain. Critical Care Nurse. 2008; 28(6): 38-49. doi: 10.4037/ccn2008.28.6.38

18. Lamont LA, Tranquilli WJ, Grimm KA. Physiology of pain. Vet Clin North Am Small Anim Pract. 2000; 30(4): 703-728. doi: 10.1016/s0195-5616(08)70003-2

19. Apkarian A, Bushnell M, Treede R, Zubieta J. Human brain mechanisms of pain perception and regulation in health and disease. Eur J Pain. 2005; 9: 463-484. doi: 10.1016/j.ejpain.2004.11.001

20. Cole EB. Pain management: Classifying, understanding, and treating pain. Hospital Physician. 2002: 23-30.

21. Coleman D, Slingsby L. Attitudes of veterinary nurses to the assessment of pain and the use of pain scales. Vet Rec. 2007; 160(16): 541-544. doi: 10.1136/vr.160.16.541

22. Lamont LA. Multimodal pain management in veterinary medicine: the physiologic basis of pharmacologic therapies. Vet Clin North Am Small Anim Pract. 2008; 38(6): 1173-1186. doi: 10.1016/j. cvsm.2008.06.005

23. Haanpää ML, Treede RD. Diagnosis and classification of neuropathic pain. Pain: Clinical Update. 2010; 18(7): 1-6.

24. Chapman CR, Nakamura Y. A passion of the soul: An introduction to pain for consciousness researchers. Consciousness and Cognition. 1999; 8(4): 391-422. doi: 10.1006/ccog.1999.0411

25. Cox F. Basic principles of pain management: assessment and intervention. Nurs Stand. 2010; 25(1): 36-39. doi: 10.7748/ ns2010.09.25.1.36.c7983

26. Hellyer P, Rodan I, Brunt J, Downing R, Hagedorn JE, Rob- 
ertson. AAHA/AAFP pain management guidelines for dogs and cats. J Feline Med Surg. 2007; 9(6): 466-480. doi: 10.1016/j. jfms.2007.09.001

27. Rayment C, Bennett MI. Definition and assessment of chronic pain in advanced disease. Oxf Textb Palliat Med. 2015: 519-524. doi: $10.1093 / \mathrm{med} / 9780199656097.003 .0093$

28. Hansen BD. Assessment of pain in dogs. Vet Clin Stud. IL AR J. 2003; 44(3): 197-205. doi: 10.1093/ilar.44.3.197

29. Bufalari A, Adami C, Angeli G, Short C. Pain assessment in animals. Vet Res Commun. 2007; 31: 55-58. doi: 10.1007/s11259. 007-0084-6

30. Flecknell PA, Waterman-Pearson A. Pain Management in Animals. Philadelphia, USA: WB Saunders; 2000.

31. Robertson SA, Sanchez LC. Clinical application of equine analgesics. Equine Pharmacology. 2015; 63-75. doi: 10.1002/9781118845110. ch4

32. Dunckley P, Wise RG, Fairhurst M, Hobden P, Aziz Q, Chang $\mathrm{L}$, et al. A comparison of visceral and somatic pain processing in the human brainstem using functional magnetic resonance imaging. J Neurosci. 2005; 25(32): 7333-7341. doi: 10.1523/JNEUROSCI.1100-05.2005

33. Barnett J. Measuring pain in animals. Aust Vet J. 1997; 75(12): 878-879. doi: 10.1111/j.1751-0813.1997.tb11256.x

34. Duncan IJ, Molony V. Assessing pain in farm animals. Directorate-General for Agriculture, Coordination of Agricultural Research; 1986.

35. Ashley F, Waterman-Pearson A, Whay H. Behavioural assessment of pain in horses and donkeys: Application to clinical practice and future studies. Equine Vet J. 2005; 37(6): 565-575. doi: 10.2746/042516405775314826

36. Stock ML, Baldridge SL, Griffin D, Coetzee JF. Bovine dehorning: Assessing pain and providing analgesic management. Vet Clin North Am Food Anim Pract. 2013; 29(1): 103-133. doi: 10.1016/j. cvfa.2012.11.001

37. Zayan R. Assessment of pain in animals: Epistemological comments. Paper presented at: Assessing pain in farm animals, proceedings of a workshop; 1986; Roslin, Scotland.

38. Egger CM, Love L, Doherty T. Pain Management in Veterinary Practice. NJ, USA: John Wiley \& Sons; 2013.

39. Kopf A, Patel NB. Guide to pain management in low-resource settings. 2010: 13.

40. Hartrick CT. Multimodal postoperative pain management. Am J Health Syst Pharm. 2004; 61(suppl_1): S4-S10. doi: 10.1093/ ajhp/61.suppl_1.S4
41. Stasiak KL, Maul D, French E, Hellyer PW, Vandewoude S. Species-specific assessment of pain in laboratory animals. Contemp Top Lab Anim Sci. 2003; 42(4): 13-20.

42. Wagner AE. Effects of stress on pain in horses and incorporating pain scales for equine practice. Vet Clin North Am Equine Pract. 2010; 26(3): 481-492. doi: 10.1016/j.cveq.2010.07.001

43. Morton CM, Reid J, Scott EM, Holton LL, Nolan AM. Application of a scaling model to establish and validate an interval level pain scale for assessment of acute pain in dogs. Am J Vet Res. 2005; 66(12): 2154-2166. doi: 10.2460/ajvr.2005.66.2154

44. Gleerup KB, Andersen PH, Munksgaard L, Forkman B. Pain evaluation in dairy cattle. Appl Anim Behav Sci. 2015; 171: 25-32. doi: 10.1016/j.applanim.2015.08.023

45. Reid J, Scott M, Nolan A, Wiseman-Orr L. Pain assessment in animals. In Practice. 2013; 35(2): 51-56.

46. Cambridge AJ, Tobias KM, Newberry RC, Sarkar DK. Subjective and objective measurements of postoperative pain in cats. J Am Vety Med Assoc. 2000; 217(5): 685-690. doi: 10.2460/ javma.2000.217.685

47. Potter R. Chronic non-malignant pain: Time to take on the challenge. J R Coll Gen Pract. 1989; 39(329): 486-487.

48. Cohen SP, Mao J. Neuropathic pain: Mechanisms and their clinical implications. BMJ. 2014; 348: f7656. doi: 10.1136/bmj.f7656

49. Cox F. An overview of pharmacology and acute pain: Part two. Nurs Stand. 2010; 25(5): 35-39. doi: 10.7748/ns2010.10.25.5.35. c8022

50. Koneru A, Satyanarayana S, Rizwan S. Endogenous opioids: Their physiological role and receptors. Global J Pharmacol. 2009; 3(3): 149-153.

51. Harirforoosh S, Jamali F. Renal adverse effects of nonsteroidal anti-inflammatory drugs. Expert Opin Drug Saf. 2009; 8(6): 669-681. doi: $10.1517 / 14740330903311023$

52. Byers M, Bonica J. Peripheral pain mechanisms and nociceptor plasticity. Bonica's Management of Pain. 2001; 3: 26-72.

53. Patil VRS, Campbell CJ, Yun YH, Slack SM, Goetz DJ. Particle diameter influences adhesion under flow. Biophys J. 2001; 80(4): 1733-1743. doi: 10.1016/s0006-3495(01)76144-9

54. Ward CW. Non-pharmacologic methods of postoperative pain management. Med Surg Nursing. 2016; 25: 9-10.

55. El Geziry A, Toble Y, Al Kadhi F, Pervaiz M, Al Nobani M. Non-pharmacological pain management. IntechOpen. 2018. doi: 10.5772/intechopen.79689 